\title{
Adenocarcinoma de uraco. Análisis de 8 años en un centro hospitalario de tercer nivel de atención
}

\author{
Urachal adenocarcinoma. Analysis of 8 years in a third level medical facility
}

Jorge Reyes-Arroyo, Laura C. Mejía-Ríos, Elieser Fernández-Vivar, Claudia T. Gutiérrez-Quiroz, Alejandro Priego-Niño y Álvaro J. Montiel-Jarquín*

Unidad Médica de Alta Especialidad, Hospital de Especialidades de Puebla, Centro Médico Nacional General de División Manuel Ávila Camàcho, Instituto Mexicano del Seguro Social, Puebla, México

\section{Resumen}

Antecedentes: El cáncer de uraco es una de las enfermedades malignas de la vejiga más raras y agresivas. Su incidencia en 2012 era del $0.2 \%$ de todos los cánceres de vejiga. Se presenta en estadios avanzados, con una mortalidad del $58 \%$ a 22 meses para el estadio IV. Se origina a partir del uraco, una estructura embriológica que está presente en el 32\% de la población adulta. Objetivo: Presentar las características clínicas de una serie de casos de cáncer de uraco en una uñidad de tercer nivel de atención médica. Método: Serie de casos. Se analizaron los resultados clínicos obtenidos de pacientes con cáncer de uraco de 2011 a 2019. Las variables incluidas fueron edad, sexo, tabaquismo, estadio clínico, tratamiento y èvolución. Se utilizó estadística descriptiva, con medidas de tendencia central y dispersión. Resultados: Fueron siete pacientes, tres hombres y cuatro mujeres, con una edad media de 54.4 años. El tipo histológico más frecuente fue adenocarcinomătipo mucinoso. Se realizó cistectomía parcial con linfadenectomía pélvica bilateral a tres pacientes (42.8\%), y cistectomía radical más linfadenectomía pélvica bilateral en los otros cuatro (57.2\%). Conclusiones: El cáncer de uraco es una enfermedad łrara. Sus manifestaciones son tardías y el signo clínico principal es la hematuria. El pronóstico es malo a mediano plazo.

Palabras clave: Carcinoma de uraco. Cistectomía. Linfadenectomía. Quimioterapia. Radioterapia.

\begin{abstract}
Background: Urachal cancer is one of the rarest and aggressive malignant diseases of the bladder; its incidence was $0,2 \%$ of all bladder cancers in 2012. It occurs in advanced stages, among stage IV tumors $58 \%$ die at 22 months. It is originated from urachal, an embryological structure that is present in $32 \%$ of the adult population. Objective: To present a case series of urachal cancer in a third level medical facility. Method: Case series. Clinical data from patients with of urachal cancer during 2011 to 2019 were analyzed. The variables included were age, gender, smoking, clinical stage, treatment and evolưtion. Descriptive statistics, measures of central tendency and dispersion were used. Results: There were seven patients; threemen and four women; the mean age was 54.4 years. The most frequent histological type was mucinous adenocarcinoma. Partial cystectomy was performed with bilateral pelvic lymphadenectomy in three (42.8\%) patients, radical cystectomy plus bilateral pelvic lymphadenectomy in the remaining four (57.2\% patients). Conclusions: Urachal cancer is rare, clinical manifestations occur late. Hematuria is the most frequent clinical sign. The medium term prognosis is bad.

Key words: Urachal carcinoma. Cystectomy. Lymphadenectomy. Chemotherapy. Radiotherapy.

Correspondencia:

Álvaro J. Montiel-Jarquín

2 norte, 2004

Centro

Fecha de recepción: 24-09-2019

C.P. 72000 , Puebla, Pue., México

E-mail: jorge22_ra@ hotmail.com

DOI: $10.24875 / C I R U .19001597$

0009-7411/@ 2020 Academia Mexicana de Cirugía. Publicado por Permanyer. Este es un artículo open access bajo la licencia CC BY-NC-ND (http://creativecommons.org/licenses/by-nc-nd/4.0/).
\end{abstract}




\section{Introducción}

El cáncer de vejiga es el séptimo cáncer diagnosticado con mayor frecuencia en la población masculina de todo el mundo. Su incidencia es del 9.0\% para los hombres y del $2.2 \%$ para las mujeres, y la mortalidad es del $3.2 \%$ para los hombres y del $0.9 \%$ para las mujeres ${ }^{1,2}$.

El cáncer de uraco es uno de los cánceres más raros de la vejiga ${ }^{3}$ : representa el $0.01 \%$ de todos los tumores malignos, el $0.7 \%$ de los cánceres de vejiga $y$ el $35 \%$ de los adenocarcinomas vesicales. Debido a que su presentación es generalmente en estadios avanzados, su pronóstico es malo a plazo corto ${ }^{3,4}$. Se origina a partir del uraco, una estructura proveniente de la alantoides, que pasa desde la cúpula vesical hasta el ombligo, presente durante el desarrollo embrionario, que involuciona a partir del tercer trimestre de la gestación, pero que puede persistir en un 32\% de la población adulta ${ }^{5-7}$. Se constituye de tres capas histológicas: capa externa de músculo liso, intermedia de tejido conectivo submucoso e interna de tipo luminal. De las anteriores, las células epiteliales que constituyen cada capa dan lugar al carcinoma de uraco $^{8}$. El $90 \%$ de los carcinomas de uraco son adenocarcinomas ${ }^{9,10}$, tienen predominio por el sexo masculino, con una relación 5:1, y suelen aparecer entre la quinta y la sexta décadas de la vida. Su localización característica es en la cúpula vesical ${ }^{10,11}$.

El pronóstico depende de la etapa clínica en que se encuentre al momento del diagnóstico; cuando el tumor se limita al uraco y la vejiga (estadios I y II), la supervivencia global a 10 años es del $95 \%$, y para la enfermedad metastásica la supervivencia global es inferior a 1 año. Estos datos hacen que el tratamiento del cáncer de uraco deba de ser agresivo $0^{9-12}$.

El objetivo de este trabajo de investigación es informar sobre la forma de presentación, el tratamiento y la evolución del cáncer de uraco en un hospital de tercer nivel de atención médica.

\section{Método}

Serie de casos. Se revisaron los expedientes clínicos de pacientes con cáncer de uraco durante el periodo comprendido de 2011 a 2019. Se incluyeron pacientes con cáncer de uraco confirmado por histopatología, de ambos sexos y de todas las edades. Las variables consideradas fueron sexo, edad al momento del diagnóstico, consumo de tabaco, síntomas clínicos, resultado
Tabla 1. Sistema de clasificación Sheldon

- Estadio I: cáncer de uraco confinado a la mucosa uracal.

- Estadio II: invasión confinada al uraco.

- Estadio IIIA: cáncer de uraco con extensión local a la vejiga.

- Estadio IIIB: cáncer de uraco con extensión a la pared abdomînal.

- Estadio IIIC: cáncer de uraco con extensión al peritoneo.

- Estadio IIID: cáncer de uraco con extensión a otra víscera diferente de la vejiga.

- Estadio IVA: metástasis a ganglios linfáticos.

- Estadio IVB: metástasis a distancia.

histológico e inmunohistoquímico tratamiento quirúrgico

histológico e inmunohistoquímico, tratamiento quirúrgico inicial además del tratamiento adyuvante realizado supervivencia global y recurrencia.

Con respecto al consumo de tabaco, en los expedientes clínicos solo se determinó si los pacientes fumaban o no fumaban. Para la clasificación clinica se utilizó el Sistema Sheldon (Tabla 1) y para el dīagnóstico se usaron los criterios del Medical Diagnóstic Anderson Cancer Center (Tabla 2). La estadística utilizada fue descriptiva, con medidas de tendengcia central y dispersión para variables ordinales, yđ̛̣recuencias y porcentajes para variables nominales en el programa estadístico SPSS v. 23 para Windoows. Este trabajo cumple con los criterios éticos en materria de investigación: valor, tiene importancia científica y clínica; validez científica, es original, idóneo y rẻalizable; los investigadores tienen experiencia en el tema; selección equitativa del sujeto, todos cumplieron con los criterios de selección; proporción favorable de riesgo-beneficio; evaluación independientên no hay conflicto de intereses ni financiamiento para la realización del trabajo; no requirió carta de consentimiento informado debido a que es un estudio derevisión de expedientes; y respecto a los sujettos inscritos, se respetan su autonomía y anonimato, El protocolo fue debidamente registrado ante el combité de investigación de la unidad médica participante.

\section{Resultados}

Hubo siete pacientes con cáncer de uraco en el periodo de estudio. La edad media fue de 54.42 año con una mínima de 35 y una máxima de 70 , y con una desviación estándar de 13.15 años. De ellos, 4 $(57.2 \%)$ eran mujeres y $3(42.8 \%)$ eran hombres;; 4 $(57.2 \%)$ fumaron y $3(42.8 \%)$ no fumaron. El síntoma predominante fue hematuria en todos los casos, seguida de mucinuria en 5 (71.4\%), dolor y tumor umbilical en $3(42.8 \%)$. 
Tabla 2. Criterios para el diagnóstico de cáncer de uraco del M.D. Anderson Cancer Center14

Criterios principales:

Ubicación en la cúpula de la vejiga o en otro lugar en la línea media de la vejiga

Demarcación aguda entre tumor y epitelio superficial normal

Criterios de apoyo:

Histología de tipo entérico

Ausencia de displasia urotelial

De acuerdo con la clasificación de Sheldon, 1 (14.3\%) paciente fue II, 4 (57.2\%) fue IIIA, 1 (14.3\%) fue IIIB y 1 (14.3\%) fue IV. Con respecto al tipo histológico, todos los pacientes fueron adenocarcinomas; de estos, 4 (57.2\%) fueron del subtipo mucinoso y los $3(42.8 \%)$ restantes del tipo no mucinoso. Todos los pacientes resultaron positivos a citoqueratina $7 \mathrm{y}$ citoqueratina 20 (Tabla 3), y $2(28.6 \%)$ pacientes para antígeno carcinoembrionario. Los resultados histopatológicos de adenocarcinoma de uraco se muestran en las figuras 1 y 2 .

A los siete pacientes se les realizó tratamiento quirúrgico: a $3(42.8 \%)$ cistectomía parcial con onfalectomía y linfadenectomía pélvica bilateral, y a 4 (57.2\%) cistectomía radical con onfalectomía y linfadenectomía pélvica bilateral.

El tratamiento adyuvante se otorgó a seis pacientes: a $4(66.6 \%)$ quimioterapia y a $2(33.4 \%)$ radioterapia.

Al momento del cierre del estudio (año 2019) se tuvo recurrencia de la enfermedad en $2(28.6 \%)$ pacientes; en 1 (14.3\%) fue en el hígado a 5 años y en el otro fue una recurrencia local a 4 años desde el inicio de la enfermedad (Tabla 4).

Se reporta una supervivencia global de 72 meses en dos de los pacientes de esta serie, de 64 meses en uno, de 24 meses en uno, de 6 meses en uno y de 4 meses en dos (Fig. 3).

\section{Discusión}

El cáncer de uraco es una enfermedad poco común y su pronóstico es malo a plazo corto. Actualmente hay menos de 400 casos informados en la literatura médica en inglés, de los cuales la mayoría corresponden a reportes de casos y revisiones de la literatura ${ }^{2,11-13}$.

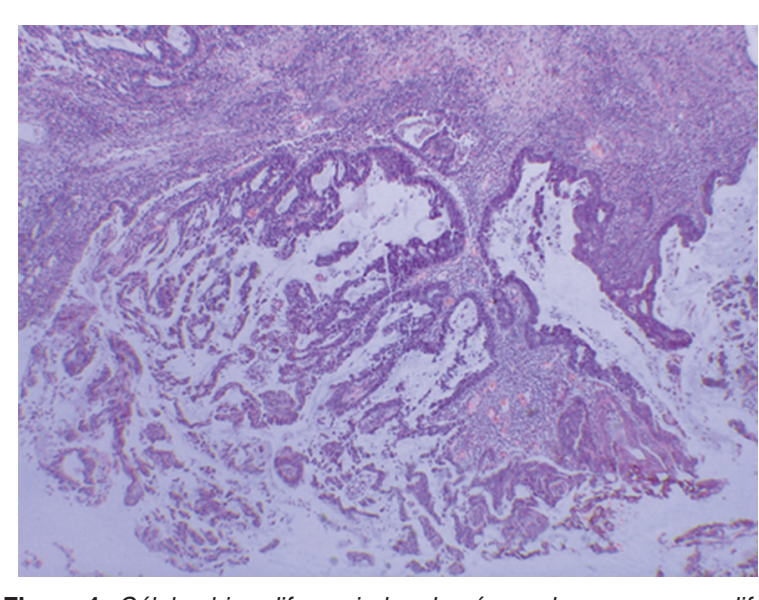

Figura 1. Células bien diferenciadas de cáncer de uraco con proliferación glandular (10x).

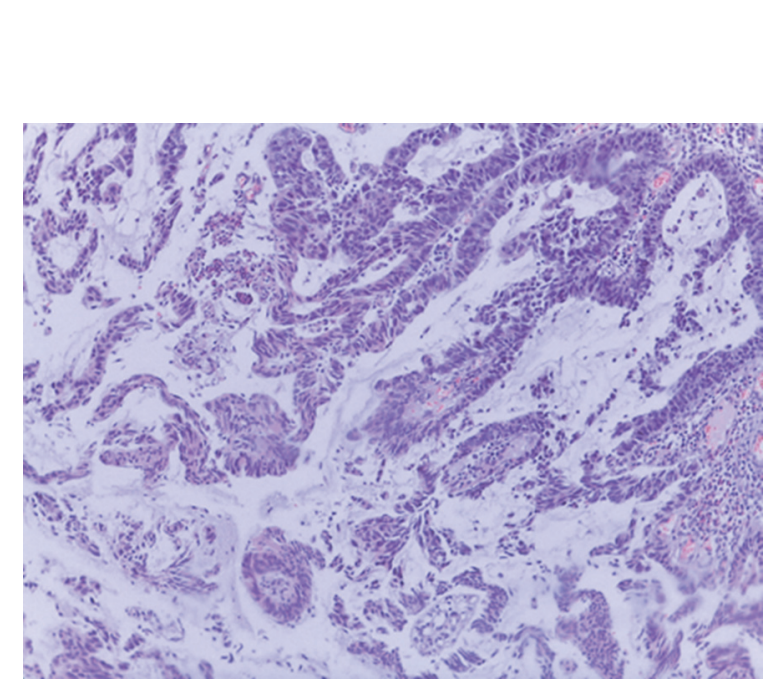

Figura 2. Proliferación glandular con células bien diferenciadas (10x).

La edad de presentación en esta serie fue en la sexta década de la vida (54.42 años), lo cual concuierda con la literatura mundial, que menciona quě la edad de presentación de estos tumores es entrè la quinta y la sexta décadas de la vida ${ }^{2,3,10,14}$. Se presenta con mayor frecuencia en los hombres que en mujeres, con una relación $5: 1$, y la incidencia esodel $9.0 \%$ para los hombres y del $2.2 \%$ para las mujeres $^{8,14-19}$, lo cual difiere de los resultados de este estudio, en el que se encontró predominio en mujeres.

En la literatura mundial, algunos autores informan una relación entre el consumo de tabaco y el desarrollo de cáncer de uraco, en tanto otros autores afirman que fumar no conduce a su desarrollo9,11,18. En esta sërie, la mayor parte de los pacientes que tuvieron consumo de tabaco fueron hombres; sin embargo, no se püdo determinar la influencia del consumo de tabaco sobre 


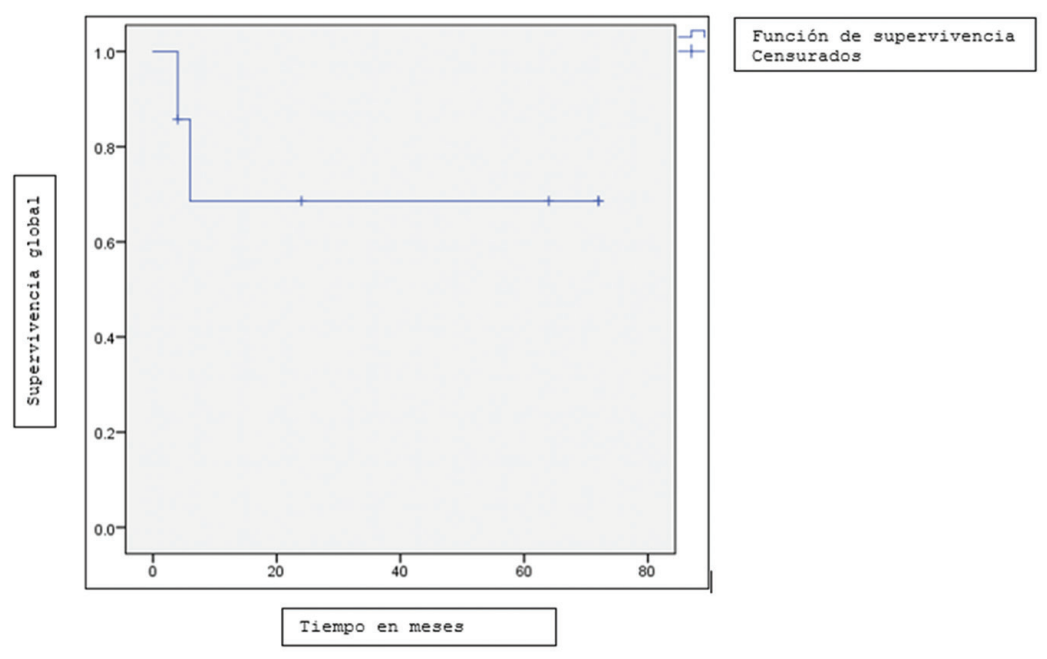

Figura 3. Supervivencia global de la población incluida en el estudio.

Tabla 3. Casos de cáncer de uraco

\begin{tabular}{|c|c|c|c|c|c|c|c|c|}
\hline Caso & Sexo & Edad & Tabaquismo & Síntomas & Sheldon & Histología & CK7, CK20 & $\underline{A C E}$ \\
\hline 1 & M & 43 & - & Hematuria, mucinuria, disuria, dolor, tumor & $\| I A$ & Mucinoso & + & ○一- \\
\hline 2 & M & 60 & + & Hematuria, mucinuria & $\|$ & No mucinoso & + & + \\
\hline 3 & M & 48 & - & Hematuria, disuria, dolor, tumor & $\| \mathrm{A}$ & No mucinoso & + & 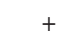 \\
\hline 4 & $\mathrm{H}$ & 56 & + & Hematuria, mucinuria & IV & Mucinoso & + & 言- \\
\hline 5 & $\mathrm{H}$ & 35 & + & Hematuria, disuria, dolor, tumor & $\| \mathrm{A}$ & $\begin{array}{l}\text { Mucinoso con células en } \\
\text { anillo de sello }\end{array}$ & + & $\frac{\mathrm{O}^{+}}{\mathrm{d}}$ \\
\hline 6 & $\mathrm{H}$ & 69 & + & Hematuria, mucinuria, disuria & $\| \mathrm{A}$ & No mucinoso & + & ㅁ- \\
\hline 7 & M & 70 & - & Hematuria, mucinuria & IIIB & Mucinoso & + & ㅇ- \\
\hline \multicolumn{8}{|c|}{ ACE: antígeno carcinoembrionario; CK: citoqueratina. } & 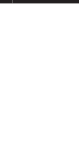 \\
\hline
\end{tabular}

Tabla 4. Tratamiento y evolución de los pacientes incluidos

\begin{tabular}{llllc}
\hline Cirugía & Qt & Rt & Recurrencia & Supervivencia \\
\hline CP + LPB & Sí & No & Hígado & 72 meses \\
CP + LPB & No & No & No & 72 meses \\
CP + LPB & No & Si & Local & 64 meses \\
CR + LPB & Sí & No & No & 4 meses $^{*}$ \\
CR + LPB & Sí & No & No & 6 meses $^{\star}$ \\
CR + LPB & No & Si & No & 24 meses $^{2}$ \\
CR + LPB & Sí & No & No & 4 meses \\
\hline
\end{tabular}

CP: cistectomía parcial; CR: cistectomía radical; LPB: linfadenectomía pélvica bilateral; Qt: quimioterapia; Rt: radioterapia.

*Paciente fallecido.

los pacientes debido a que únicamente se les investigó si fumaban o no, y en ningún expediente se determinaron la cantidad y el tiempo de consumo.
En cuanto a la presentación clínica del cánces $d e$ uraco, los síntomas son, por orden de frecuencia, hematuria macroscópica (73\%), dolor abdominal ümal localizado (14\%), disuria intermitente (13\%), mucōsuria $(10 \%)$, síntomas irritativos del tracto urinario inferior $(40 \%)$, tumor palpable en la parte baja del abdomen (17\%), bacteriuria (8\%) y flujo mucoso o sanguinolento umbilical $(2 \%)^{13,17}$. En nuestros pacientes, la presentación de los síntomas fue similar: Enematuria macroscópica en el 100\%, dolor referid en el hipogastrio de moderada intensidad en el $42.8 \%$, mucosuria en el $71.5 \%$, tumor umbilical en el $42.8 \%$ y salida de líquido mucosanguinolento a través de la

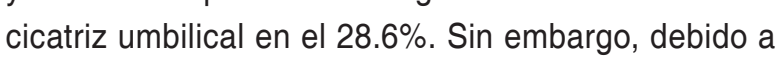
que la sintomatología se presenta en los estadios tardíos, esta es una enfermedad de diagnóstico taŕdío y pronóstico malo. 
Además de los criterios del M.D. Anderson Cancer Center (Tabla 2), para el diagnóstico de cáncer de uraco utilizamos la cistoscopía con toma de biopsia del tumor vesical como estudio diagnóstico fundamental, el cual nos da una orientación para su estadificación clínica debido a la posibilidad de ubicar la lesión en el interior de la vejiga ${ }^{18,19}$.

La tomografía computarizada es el estudio de imagen más importante para el diagnóstico del carcinoma de uraco, ya que además se utiliza para la estadificación clínica de la enfermedad y nos da una imagen sobre la extensión local y sobre el estado de los nódulos linfáticos y la posible extensión a distancia. Uno de los signos considerados patognomónicos del cáncer de uraco es la presencia de tumor en la línea media de la vejiga y de calcificaciones periféricas en el tumor ${ }^{14,18}$. El $100 \%$ de los pacientes fueron evaluados con tomografía computarizada.

La estadificación de los pacientes al momento del diagnóstico fue en etapas avanzadas, el $71.4 \%$ Sheldon III y el $14.2 \%$ Sheldon IV, lo cual coincide con la literatura y se debe a que la enfermedad es asintomática en las etapas iniciales. Solo se encontró el $14.28 \%$ de los pacientes en Sheldon II.

En cuanto a la clasificación histológica, el adenocarcinoma de uraco se divide en cinco subtipos: intestinal, mucinoso, de células en anillo de sello, mixto e inespecífico ${ }^{11,13}$. En este estudio todos los pacientes fueron del tipo adenocarcinoma, y en cuanto al subtipo, el más frecuente fue el mucinoso, con un $57.2 \%$, seguido del no mucinoso con un $42.8 \% \%^{3,6,11,13}$.

En la literatura consultada, la inmunohistoquímica para citoqueratina 7 y 20 fue positiva en el $60 \%$ de los casos de adenocarcinoma de uraco. La negatividad de la inmunohistoquímica para la citoqueratina 7 favorece el desarrollo de cáncer colónico en estos pacientes ${ }^{4,20,21}$. En este estudio, el resultado de la inmunohistoquímica fue positivo para citoqueratina 7 y 20 en todos los pacientes. Además, el antígeno carcinoembrionario fue positivo en el $28.6 \%$ de los pacientes.

El tratamiento de referencia del cáncer de uraco no metastásico es el quirúrgico y se basa en el estadio clínico al momento del diagnóstico; puede ser cistectomía parcial o radical, más onfalectomía y linfadenectomía pélvica bilateral, teniendo en cuenta que en los hombres se debe realizar prostatectomía radi$\mathrm{cal}^{4,9,10,15}$. En esta serie, al $42.8 \%$ de los pacientes se les realizó cistectomía parcial y al $57.2 \%$ radical, más onfalectomía-linfadenectomía pélvica bilateral y prostatectomía radical en los hombres (Tabla 3).
Actualmente, el papel de la quimioterapia y de la radioterapia adyuvante no está del todo claro, p̄ero existen estudios que demuestran respuesta en allgunos $\operatorname{casos}^{12}$.

El tratamiento adyuvante con quimioterapia se băsa en los esquemas de cisplatino + paclitaxel + ifosfamida, 5-fluorouracilo + doxorubicina, metotrexato + 5-fluorouracilo + epirubicina + cisplatino, irinotecán, y paclitaxel + metotrexato + cisplatino. Sin embargo, no se hàdemostrado la efectividad de un esquema estándar ê el tratamiento del carcinoma de uraco; solo existen escasos reportes de casos con respuesta a esquemas de quimioterapia. Actualmente se están realizando estudios para valorar el uso de quimioterapia neoadyuvănte y adyuvante en el tratamiento del cáncer de uraco con el fin de tener mejores estrategias de tratamiento y্rresultados más favorables para esta enfermedad $19,22,233$.

Se inició tratamiento con quimioterapia adyuvante con esquema de cisplatino y gemcitabina en 4 (57.2\%) pacientes y en 2 (28.6\%) radioterapia con $30 \mathrm{~Gy}$ vididos en 10 fracciones.

Con respecto al pronóstico, cuando se diagnosfica como enfermedad confinada al uraco y la vejigà, la supervivencia es mayor de 10 años, pero para lacenfermedad metastásica es inferior a 1 año ${ }^{11}$.

El $28.6 \%$ de los pacientes presentó recurrenciẩ de la enfermedad; de estos, el $14.3 \%$ fue en el hígađo y el otro $14.3 \%$ fue recurrencia local a 5 y 4 años:-

Se concluye que el cáncer de uraco es una enfermedad poco frecuente, indolente, que se manifiesta tardíamente, cuyo principal signo clínico es la hematuria y tiene un pronóstico malo a plazo mediano. Al momento del cierre del estudio no es posible determinar la mortalidad a 10 años en este grupo decopacientes debido a que algunos no completan el tiempo de seguimiento.

\section{Conflicto de intereses}

Los autores declaran no tener conflicto de intereses.

\section{Responsabilidades éticas}

Protección de personas y animales. Los autores declaran que para esta investigación no se han realizado experimentos en seres humanos hi en animales.

Confidencialidad de los datos. Los autores declaran que han seguido los protocolos de su centrô de trabajo sobre la publicación de datos de pacientes. 
Derecho a la privacidad y consentimiento informado. Los autores han obtenido el consentimiento informado de los pacientes y/o sujetos referidos en el artículo. Este documento obra en poder del autor de correspondencia.

\section{Bibliografía}

1. Cruz P, Ramírez M, Iborra I, Rubio J, Pinto F, Gómez-Ferrer A. Adenocarcinoma de uraco: ¿qué tanto sabemos? Rev Mex Urol. 2014;74:301-7.

2. Martínez-Cornelio A, Flores-López D, Ojeda RF, Quintero-Becerra J Hernández-Toriz N. Experiencia quirúrgica en el adenocarcinoma de uraco. Cir Cir. 2009;77:33ロ8.

3. Donate-Moreno MJ, Giménez-Bachs JM, Salinas-Sánchez AS, Lorenzo-Romero JG, Segura-Martín M, Hernández-Millan I. Patología del uraco: revisión de conjunto y presentación de tres casos. Actas Urol Esp. 2005;29:332-6.

4. Türk $C$, Skolarikos $A$, Neisius $A$, Babjuk $M$, Burger $M$, Compérat $E$, et al. European Association of Urology 2019 guidelines on non-muscle-invasive bladder cancer. Eur Board Urol. 2019; 75(5p):e121-e146.

5. Elser C, Sweet J, Sendhil K, Masoom A, Jewett C, Sridhar S. A case of metastatic urachal adenocarcinoma treated with several different chemotherapeutic regimens. Can Urol Assoc J. 2012;6:27-31.

6. Planeles-Gómez J, Olmos-Sánchez L, Sánchez-Cuallado C, Esteban-Fernández J, De la Rosa-Martínez F, Romero-Costa M. Adenocarcinoma de uraco: presentación de dos casos. Rev Chil Urol. 2018;83:44-8.

7. Palla J, Sampaio R, Peixoto C. Urachal tumor: a case report of an extremely rare carcinoma. Case Rep Pathol. 2017;2017:1-5.

8. García-Salazar N, Pérez Silva MM, Acuña Hernández M. Carcinoma de uraco, una neoplasia maligna poco frecuente. Rev Med Urug. 2018;34:52-5.

9. Bruins HM, Visser O, Ploeg M, Hulsbergen CA, Lambertus AL, Witjes MH, et al. The clinical epidemiology of urachal carcinoma: results of a large population-based study. J Urol. 2012;188:1102-7.
10. Tibor S, Orsolya M, Niedworok C, Henning R, Szendröi A, Marcell A, et al. Clinical, prognostic, and therapeutic aspects of urachal carcinoma. A comprehensive review with meta-analysis of 1,010 cases. Urof Oncol. 2016;34:388-98.

11. Reis H, Krafft U, Niedworok C, Módos O, Herold T, Behrendt M, At-Ahmadie $\mathrm{H}$, et al. Biomarkers in Urachal Cancer and Adenocarcinomas in the Bladder: A Comprehensive Review Supplemented by Own Dafa. Dis Markers. 2018;2018:7308168. doi: 10.1155/2018/7308168.

12. Henly D, Farrow G, Zincke H. Urachal cancer: role of conservative surgery. Urology. 1993;42:635-9.

13. Molina J, Quevedo JF, Furth AF, Richardson RL, Zincke H, Burch PA. Predictors of survival from urachal cancer. A Mayo Clinic study of 49 cases. ACS. 2007;110:2434-40

14. Siefker A, Gee J, Shen Y, Wen S, Daliani D, Millikan RE, et al. Multimodality management of urachal carcinoma: the M. D. Anderson Cancer Center experience. J Urol. 2003;69:1295-8.

15. Quan J, Xiang P, Jin L, Tao H, Hu J, Shi B, et al. Urachal carciñoma: report of two cases and review of the literature. Mol Clin Oncol. 2017;6:101-4.

16. Ashley R, Inman B, Sebo T, Ashley RA, Leibovich B, Blute ML, tẹt al. Urachal carcinoma: clinicopathologic features and long-term outcomes of an aggressive malignancy. Cancer. 2006;107:712-20.

17. Sheldon C, Clayman R, González R, Williams RD, Fraley EE. Malignant urachal lesions. J Urol. 1984;131:1-8.

18. Radtke SA. Urachal carcinoma: surgical and chemotherapeutic options. Expert Rev Anticancer Ther. 2006;6:1715-21.

19. Jung H, Sun J, Park S, Know GY, Lim HY. Treatment outcome and relevance of palliative chemotherapy in urachal cancer. Chemotherapy. 2014;60:73-80.

20. Cruz P, Ramírez M, Iborra I, Rubio J, Pinto F, Gómez A, et al. Áđenocarcinoma de uraco: ¿qué tanto sabemos? Rev Mex Urol. 2014;75:301-7.

21. Siefker A. Urachal adenocarcinoma: a clinician's guide for treatment. Semin Oncol. 2012;39:619-24.

22. Sandoval-Téllez JG, Ricárdez-Espinosa AA, Suárez-Uriarte MSP Reyes-De La Garza I, Jiménez-López A. Carcinoma del uraco: reporte de un caso y revisión de la bibliografía. Rev Mex Urol. 2018;78:215-9

23. Da Cruz CL, Fernandes GL, Natal MR, Taveira TR, Bicalho PA, de Brito YQ. Urachal neoplasia: a case report. Radiol Bras. 2014;47:387ø8. 